\title{
The Effect of Fuel Staging on the Structure and Instability Characteristics of Swirl-Stabilized Flames in a Lean Premixed Multi-Nozzle Can Combustor
}

\author{
Dr. Janith Samarasinghe \\ Center for Combustion, Power and Propulsion \\ The Pennsylvania State University, University Park, PA 16802 \\ janith.samarasinghe@ge.com \\ *Currently at GE Global Research, 1 Research Circle, Niskayuna, NY 12309 \\ Wyatt Culler \\ Center for Combustion, Power and Propulsion \\ The Pennsylvania State University, University Park, PA 16802 \\ wrc5047@psu.edu \\ Dr. Bryan D. Quay \\ Center for Combustion, Power and Propulsion \\ The Pennsylvania State University, University Park, PA 16802 \\ bdq100@psu.edu \\ Dr. Domenic A. Santavicca \\ Center for Combustion, Power and Propulsion \\ The Pennsylvania State University, University Park, PA 16802 \\ das8@psu.edu \\ Dr. Jacqueline O'Connor \\ Center for Combustion, Power and Propulsion \\ The Pennsylvania State University, University Park, PA 16802 \\ jxo22@engr.psu.edu
}




\section{ABSTRACT}

Fuel staging is a commonly used strategy in the operation of gas turbine engines. In multi-nozzle combustor configurations, this is achieved by varying fuel flow rate to different nozzles. The effect of fuel staging on flame structure and self-excited instabilities is investigated in a research can combustor employing five swirl-stabilized, lean-premixed nozzles. At an operating condition where all nozzles are fueled equally and the combustor undergoes a selfexcited instability, fuel staging successfully suppresses the instability: both when overall equivalence ratio is increased by staging as well as when overall equivalence ratio is kept constant while staging. Increased fuel staging changes the distribution of time-averaged heat release rate in the regions where adjacent flames interact and reduces the amplitudes of heat release rate fluctuations in those regions. Increased fuel staging also causes a breakup in the monotonic phase behavior that is characteristic of convective disturbances that travel along a flame. In particular, heat release rate fluctuations in the middle flame and flame-flame interaction region are out-of-phase with those in the outer flames, resulting in a cancellation of the global heat release rate oscillations. The Rayleigh integral distribution within the combustor shows that during a self-excited instability, the regions of highest heat release rate fluctuation are in phasewith the combustor pressure fluctuation. When staging fuel is introduced, these regions fluctuate out-of-phase with the pressure fluctuation, further illustrating that fuel staging suppresses instabilities through a phase cancellation mechanism. 


\section{INTRODUCTION}

Increasingly stringent emissions regulations have led gas turbine manufacturers to transition from diffusion combustion to lean premixed combustion. While lean premixed combustors can significantly reduce emissions with better fuel-air mixing and lower combustion temperatures, they are more susceptible to combustion instabilities. Combustion instability is a naturally occurring feedback loop that involves interactions between acoustic oscillations, heat release rate fluctuations from the flame, and velocity and/or equivalence ratio fluctuations of the reactants. Consequences of combustion instability include enhanced heat transfer to engine components, increased mechanical loads, and flame flashback/blowoff, all of which could potentially damage engine hardware. Therefore, the presence of combustion instabilities represents a major challenge in the design and operation of low emissions gas turbine combustors.

The interaction between acoustic oscillations and heat release rate fluctuations is described using the criterion proposed by Rayleigh [1], which states that energy is added to an acoustic field if the pressure perturbations and heat release rate perturbations are in phase. Satisfying the Rayleigh criterion alone is not sufficient to ensure that the combustor is unstable. The energy added by the heat release rate fluctuations must be greater than the energy removed by damping sources, such as viscosity, heat transfer, and sound radiation. The acoustic oscillations then trigger flow oscillations; the means by which this acoustic/flow coupling occurs depends on the geometry of the system. In swirl-stabilized combustors that are commonly used in practical gas turbine engines, the flow perturbations are typically in the form of vorticity and swirl fluctuations. Additionally, the acoustic fluctuations can drive oscillations in the fuel flow rate, resulting in mixture fluctuations. 
The generation of heat release rate fluctuations by velocity and/or equivalence ratio perturbations (known as flame response) is the final part of the combustion instability feedback loop. Flame response is still not fully understood and thus is the subject of significant research. Multiple studies have been conducted over the past few decades to understand the governing mechanisms of flame response, encapsulated in review papers [2-4] that discuss the current state of this research. A number of flame response mechanisms have been proposed: the interaction of coherent ring-vortex structures with the flame $[5,6]$, swirl fluctuations that perturb the flame base $[7,8]$, and helical instabilities interacting with the flame $[9,10]$. While a consensus on governing flame response mechanisms has not yet been reached, the above studies do indicate that flame structure (overall flame shape and distribution of heat release) plays an important role in determining flame response.

A majority of flame response research has been conducted on laboratory-scale singlenozzle combustor configurations, with a limited number of studies on multi-nozzle combustor configurations. Work in both can combustors [11] and annular combustors [12] show that the flame structure in multi-nozzle combustors is very different to that in single-nozzle combustors. These variations in flame structure can be attributed to effects unique to multi-nozzle combustors such as interactions between swirling flows, flame-flame interactions, and non-axisymmetric flame-wall interactions [13]. Therefore, it is reasonable to expect that the flame response (and thus the behavior in the presence of combustion instabilities) of multi-nozzle flames would differ to that of single-nozzle flames.

Combustion instabilities can be suppressed using two methods: active control and passive control. Active control methods typically involve the use of dynamic, closed-loop control of the instability using actuators to excite acoustic waves and/or fuel flow rate oscillations [14]. 
Alternatively, passive control strategies do not attempt to control the instability on the timescales of the acoustic oscillations, but rather they typically damp the instability or de-phase the acoustic and heat release rate oscillations.

A strategy that is used both as an active and a passive control method is fuel staging (or fuel splitting) [15]. This involves varying the distribution of fuel flowing into the combustor. A commonly used method of fuel staging is modifying flow rates between different fuel circuits of a single injector [15]. In particular, pilot fuel circuits are used to add small amounts of fuel directly to the flame region, reducing the level of premixedness but aiding in flame stabilization. In combustors that employ multiple nozzles, fuel staging can also be achieved by changing the fuel flow rate to different nozzles [16]. The fuel split between separate circuits (also known as an operating mode) mainly depends on the required combustor load. For example, the GE DLN-2.6 combustor has four fuel circuits and uses 8 different fuel staging strategies for loads from ignition to full power [17]. The multiple fuel circuits create a large parameter space for both combustor load variation and instability control; in the current work, the operating space is restricted by only having two fuel circuits.

Despite fuel staging for instability control being a common practice, no literature currently exists on the mechanisms by which fuel staging suppresses instabilities. However, the need for understanding the mechanisms by which fuel staging suppresses combustion instability is vital. Experience has shown that balancing instability amplitudes and emissions output during fuel-staging operations is difficult; fuel staging typically requires that a fuel circuit (whether it be a number of nozzles or even a circuit within one nozzle) run at a higher equivalence ratio in order to ensure flame stability. This richer flame zone is a source of higher NOx production, where these engines are typically running at the limits of the emissions regulations. A more 
detailed mechanistic understanding of how fuel staging suppresses instabilities could help to improve staging strategies in industrial hardware where combustors are running in a thin margin between stability and emissions limits.

This paper presents an experimental investigation of the mechanisms by which fuel staging suppresses self-excited combustion instabilities in a multi-nozzle can combustor. Two optical measurements, line-of-sight chemiluminescence images and OH-PLIF, are used to understand the flame structure when the combustor is undergoing a combustion instability and the means by which fuel staging affects the flame structure. The insights gained from these measurements are used to identify the mechanisms by which fuel staging suppresses combustion instabilities.

\section{EXPERIMENTAL SETUP AND MEASUREMENTS}

\section{Experimental Setup}

Experiments are conducted in a five-nozzle can combustor. Details on this combustor have been presented in previous publications $[13,18]$, which can be referred to for in-depth information about the test rig. The industrial scale, swirl and bluff body stabilized nozzles are arranged in a "four-around-one" configuration with nozzle spacing relative to the diameter of the combustor can being typical of industrial combustors. The combustor liner is a $260 \mathrm{~mm}$ diameter, $300 \mathrm{~mm}$ long quartz tube that provides optical access of the flame for diagnostics. The quartz tube is open ended and thus combustion occurs at atmospheric pressure. The combustor is operated with a fully premixed (fuel and air are mixed far upstream of the nozzle) mixture of air and natural gas ( $\sim 95 \%$ methane). Fuel staging is achieved by injecting additional fuel to the middle nozzle of the four-around-one configuration. The staging fuel enters the nozzle through 
fuel injection holes at the swirler (and is thus technically premixed when it enters the combustor). Figure 1 shows a CAD model of the multi-nozzle can combustor.

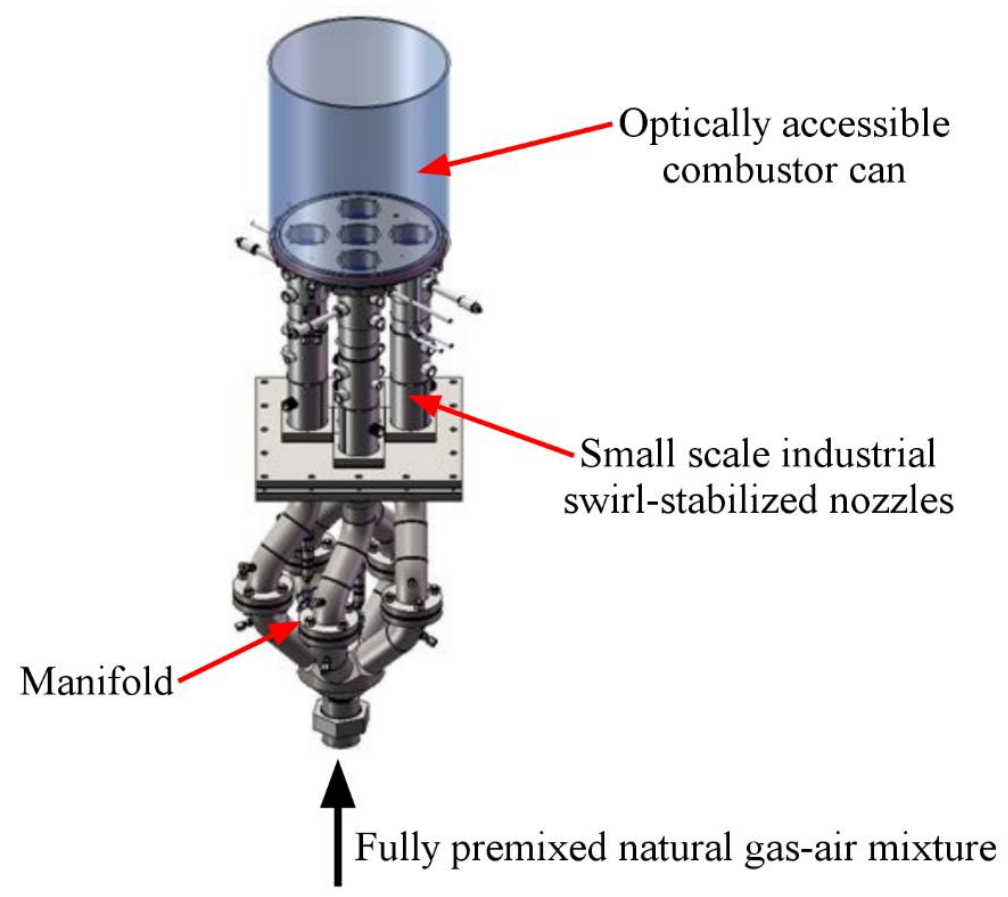

Figure 1: Schematic of Multi-Nozzle Can Combustor [13]

\section{Measurements}

Time-averaged measurements of overall fuel and air flow rates, pressure drop across each of the swirlers, and temperature of the fuel-air mixture at the inlet to the combustor are used to characterize the overall operating state of the experiment. The overall flowrates of air and fuel are measured using thermal mass flow meters. The pressure drop across each of the five swirlers is measured using electronic differential pressure gauges. The mean velocity in each nozzle is determined from the pressure drop measurements using an empirical calibration. The measured nozzle-to-nozzle variation in mean velocity is $6 \%$. Temperatures of the reactants as well as surrounding nozzle hardware (combustor dump plate and centerbodies) at the start and end of the data acquisition are measured using K-type thermocouples. 
Time-resolved measurements of combustor pressure fluctuations are obtained using a piezoelectric dynamic pressure transducer (PCB 112A22) with a built-in signal conditioner mounted flush with the dump plate. At each test case, sixteen seconds of data are acquired at an acquisition rate of $8192 \mathrm{~Hz}$.

The heat release rate from the flame is measured using $\mathrm{CH}^{*}$ chemiluminescence, a widely used indicator of heat release rate for fuel-lean premixed hydrocarbon flames [19, 20]. $\mathrm{CH}^{*}$ chemiluminescence is also used as an indicator of flame structure [19]. In the current experiment, line-of-sight $\mathrm{CH}^{*}$ chemiluminescence images obtained using a Photron SA4 highspeed camera fitted with an Invisible Vision UVi 1850-10 intensifier, a Nikon AF MicroNikkor $60 \mathrm{~mm} \mathrm{f} / 2.8 \mathrm{D}$ lens, and a $432 \mathrm{~nm} \pm 5 \mathrm{~nm}$ bandpass filter are used to characterize flame stabilization and heat release rate distribution (henceforth referred to as flame structure). At each test condition, one second of high-speed image data is acquired at 4000 frames-per second. Figure 2 shows a digital photograph and a time-averaged chemiluminescence image of the flame from the same line-of-sight (flow direction is bottom to top). This and all subsequent chemiluminescence images are presented in pseudo-color. 


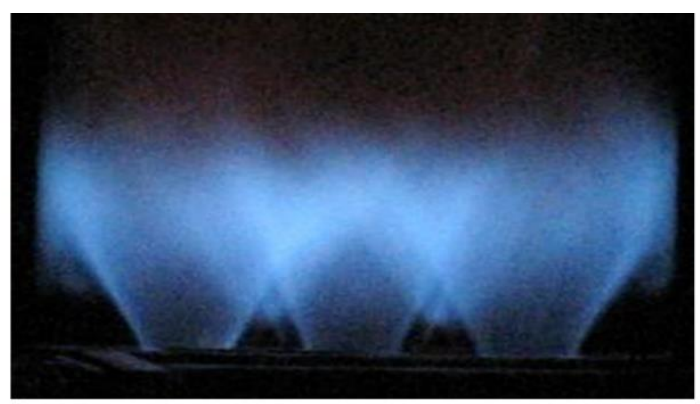

(a)

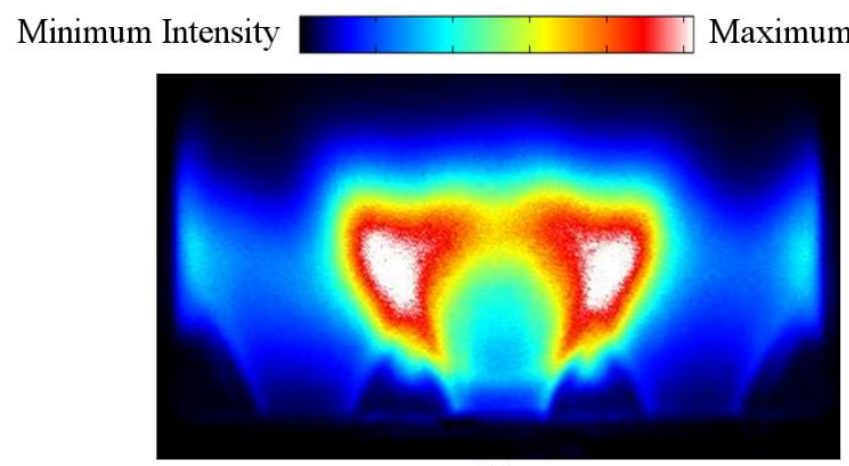

(b)

Figure 2: Line-of-sight (a) Digital photograph and (b) time-averaged chemiluminescence image of a stable multi-nozzle flame

The image captures the summed chemiluminescence emission from the entire flame along the line-of-sight, and in this particular view, the summed chemiluminescence from three flames is being captured in the middle part of the image, making it more intense than the outer flames. The most intense chemiluminescence is observed at the locations where adjacent flames interact and this has been attributed to increased flame wrinkling caused by intense turbulence in those regions. In premixed flames, flame wrinkling increases the local rate of heat release rate, which manifests as increased chemiluminescence.

The instantaneous location of the flame sheet is determined using time-resolved $\mathrm{OH}-$ PLIF measurements. The OH-PLIF system consists of a $532 \mathrm{~nm}$ wavelength Nd:YAG laser (Edgewave) which is used to pump a high-speed Sirah Credo dye laser which is tuned to produce a beam wavelength of $566 \mathrm{~nm}$ which is then frequency doubled using a BBO crystal to a 
wavelength of $283 \mathrm{~nm}$. The laser is operated at a pulse rate of $5 \mathrm{kHz}$ and the pulse energy at the dye laser aperture is approximately $0.17 \mathrm{~mJ}$ per-pulse. The beam is expanded to a height of 16 $\mathrm{mm}$ and focused to a thin sheet using a LaVision sheet forming optic. OH-PLIF images are captured at a rate of $5 \mathrm{kHz}$ using a Photron SA5 high-speed camera fitted with an intensifier (LaVision high-speed Intensified Relay Optic), a UV camera lens (Nikon UV-Nikkor f4.5 $105 \mathrm{~mm})$ and an $\mathrm{OH}$ filter $(320 \mathrm{~nm} \pm 25 \mathrm{~nm})$. The OH-PLIF images have a resolution of 5 pixels/mm. Figure 3a shows a schematic of the OH-PLIF setup.

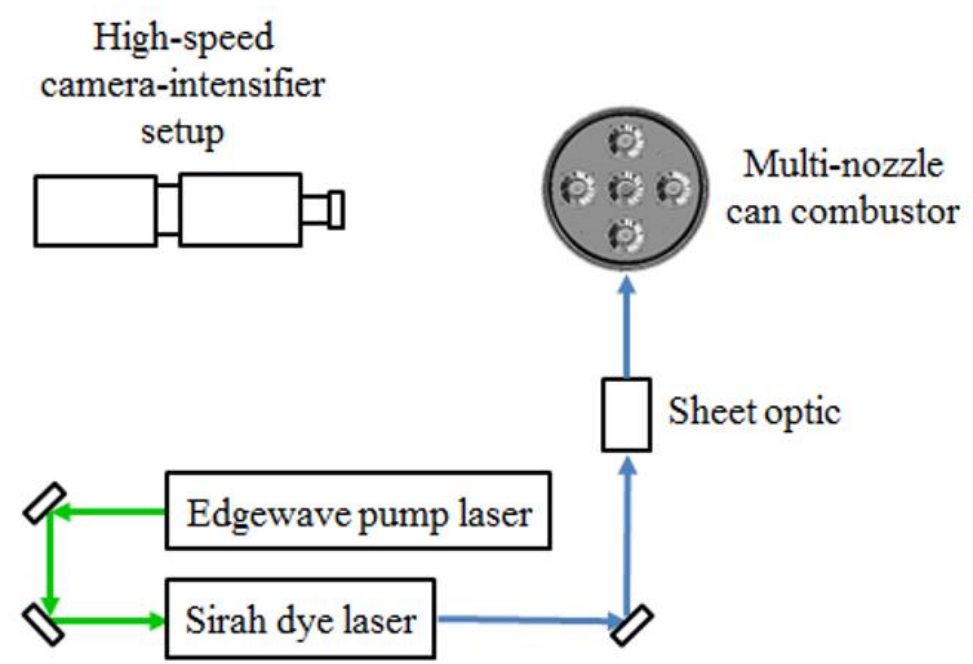

(a)

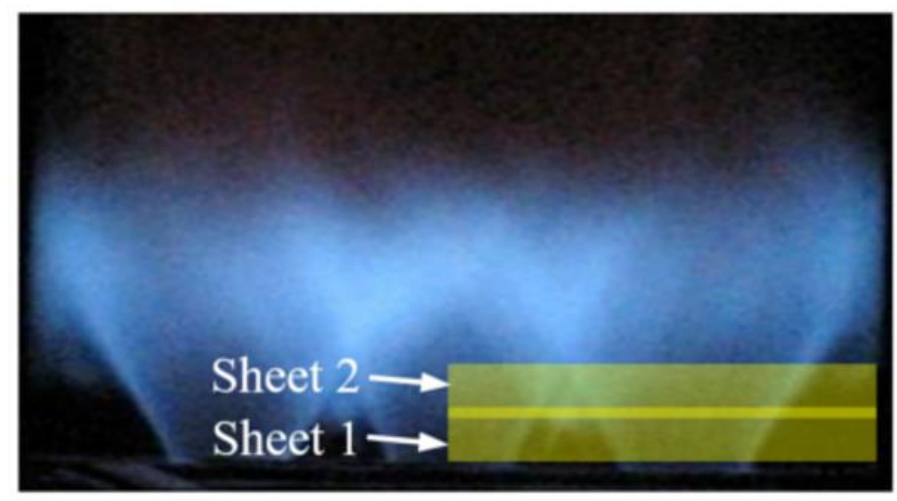

(b)

Figure 3: (a) OH-PLIF setup and (b) line-of-sight photograph of the multi-nozzle flame with laser sheets highlighted 
The current OH-PLIF setup allows an imaging region that encompasses a single outer flame and half of the middle flame. However, the OH-PLIF system was not powerful enough to illuminate the full downstream extent of the multi-nozzle flame with adequate signal. To overcome this OH-PLIF data were obtained separately at two different laser sheet heights with a $2 \mathrm{~mm}$ overlap region. Calibrating the images from the two laser sheet heights to a common coordinate system allows the mean and phase-averaged images to be stitched together. The overlapping region between the stitched images comprises the average of the intensities of the upper and lower images. The image stitching process causes faint horizontal lines at these locations. Figure $3 \mathrm{~b}$ shows a photograph of the multi-nozzle flame with the two PLIF imaging regions highlighted. All the OH-PLIF images were corrected for Gaussian beam intensity distribution before post-processing.

\section{Data processing}

Combustor pressure fluctuation is measured as a discrete time-varying signal, which is then analyzed in the frequency domain to characterize the pressure fluctuation amplitude. To calculate the instability amplitude, we first calculate the single-sided power spectrum density of the signal's linear spectrum. A high-pass filter at $10 \mathrm{~Hz}$ is then applied to the linear spectrum to remove noise due to mean drift of the pressure transducer. The root mean square (RMS) amplitude is then obtained by applying Parseval's theorem [21] to the filtered linear spectrum.

In order to study the unsteady flame structure, chemiluminescence images are processed in the frequency domain to characterize the variation of flame structure at the dominant instability frequency. The steps for this process are as follows:

1. Each pixel of the high-speed image is treated as an independent time series and its linear spectrum obtained using a fast-Fourier transform. 
2. All frequency components except for $\mathrm{a} \pm 5 \mathrm{~Hz}$ band centered at the dominant instability frequency (obtained from the linear spectrum of combustor pressure fluctuation) are zeroed to obtain a filtered linear spectrum.

3. By applying Parseval's theorem to the single-sided power spectral density of the filtered linear spectrum, the RMS component is obtained.

4. The phase relative to the start of data acquisition is the inverse tangent of the ratio of the imaginary and real components of the linear spectrum at the instability frequency.

5. The mean component is simply the average of all 4000 image frames acquired.

The above process is applied to all pixels in an acquired high-speed image set, resulting in a mean, RMS, and phase image, where the RMS and phase images are calculated at the instability frequency. The mean images provide information about the time-averaged distribution of heat release within the flame, RMS images can be used to identify the locations of highest heat release rate fluctuations, and the variation in phase in the phase images can be used to identify governing mechanisms and the coherence of the instability.

Additionally, a phase-averaged time series for a single instability cycle can be generated using the mean $(\bar{a})$, RMS $\left(a_{R M S}^{\prime}\right)$, and phase ( $\left.a_{\text {phase }}\right)$ data obtained above by inputting them to Eq. (1) below:

$$
a(t)=\bar{a}+\sqrt{2} a_{R M S}^{\prime} \cos \left(2 \pi f t+a_{\text {phase }}\right)
$$

where $f$ is the instability frequency and $t$ is the time during a single instability cycle. For all phase-averaged data presented in this paper, the time increment was set such that each instability cycle contained 24 data points.

The detailed flame structure of the base of the multi-nozzle flame is characterized using time-averaged flame surface density (FSD) obtained from the OH-PLIF data. Raw OH-PLIF 
images are first smoothed using a $5 \times 5$ pixel bilateral filter before converting the images to binary using an intensity threshold (i.e., values above the threshold are assigned a value of one to denote burnt gases and values below the threshold are assigned a value of zero to denote reactants). Edges in each image frame are identified from the binary images using the MATLAB function bwboundaries. The FSD is then computed by calculating the flame length within a 9x9 pixel square interrogation window moved along the edges. The frames are then averaged to obtain a time-averaged FSD image. In this paper, FSD is used as a qualitative marker of flame location and flame area.

Mapping the distribution of the Rayleigh integral, which is the mathematical description of the Rayleigh criterion, within the combustor provides insight about which regions of the flame are most likely to drive an instability and the means by which fuel staging may alter the behavior of these regions. This "local" Rayleigh integral is defined in Eq. (2) as the product of the fluctuating components of pressure $\left(p^{\prime}\right)$ and heat release rate $\left(q^{\prime}\right)$ at a specified location within the combustor $(\boldsymbol{x})$ integrated over a single cycle of the instability $(T)$.

$$
R I(\boldsymbol{x})=\int_{T} p^{\prime}(\boldsymbol{x}, t) q^{\prime}(\boldsymbol{x}, t) d t
$$

In this paper, the variation in pressure fluctuation amplitude along the flow direction within the combustor $p^{\prime}(x, t)$ is modeled using Eq. (3) where $p^{\prime}(0, t)$ is the measured dump plate pressure fluctuation filtered at the instability frequency $(f)$, and $c$ is the speed of sound at the recirculation zone temperature.

$$
p^{\prime}(x, t)=p^{\prime}(0, t) \cos \left(\frac{2 \pi f x}{c}\right)
$$

Line-of-sight chemiluminescence images that are filtered at the instability frequency are used to characterize the local heat release rate fluctuations. Due to the line-of-sight nature of the 
measurement, the chemiluminescence intensity is integrated along the depth of the combustor. The local Rayleigh integral obtained from the line-of-sight images is:

$$
R I(x, y)=\int_{T} p^{\prime}(x, t) q^{\prime}(x, y, t) d t
$$

Where $\mathrm{x}$ is the coordinate of the image along the combustor centerline and $\mathrm{y}$ is the coordinate of the image along the combustor diameter. In all instances where the Rayleigh integral distribution is calculated in this paper, both the pressure fluctuation and chemiluminescence fluctuation data are phase-averaged using Eq. (1).

\section{Ensuring repeatability of the data points}

Previous studies of combustion instabilities show that thermal boundary conditions can affect the amplitude of combustor pressure fluctuations [22]. This is confirmed for the current combustor, where Fig. 4 shows the variation in RMS amplitude of combustor pressure fluctuation with dump plate and centerbody temperatures. The inlet conditions (velocity, equivalence ratio, and temperature) were kept constant at all ten data points in Fig. 4 and the combustor is subject to a self-excited instability at this operating condition. Each data point consists of 16 seconds of combustor pressure fluctuation data which is divided into 1 second sets. The points in the plot are the means of the 16 data sets and the error bars denote \pm 1 standard deviation. Both the amplitude and the standard deviation of pressure fluctuation amplitudes increase at the highest centerbody and dump plate temperatures.

In order to ensure repeatability and reproducibility of data, the combustor is first operated at a stable (no self-excited instability) baseline condition for a period of time sufficiently long to ensure that the dump plate and centerbody temperatures stabilize. Then the desired operating condition is reached and data are acquired. Following data acquisition, the combustor is run at a 
fuel lean, stable condition close to lean blowoff to cool the hardware. Before the next data acquisition, the combustor is once again operated at the baseline operating condition until the dump plate and centerbody temperatures reach their baseline values. This operating procedure ensures that the centerbody and dump plate temperatures at each data point acquired are within a $5 \mathrm{~K}$ range of the target condition, thus ensuring that all measurements are repeatable.

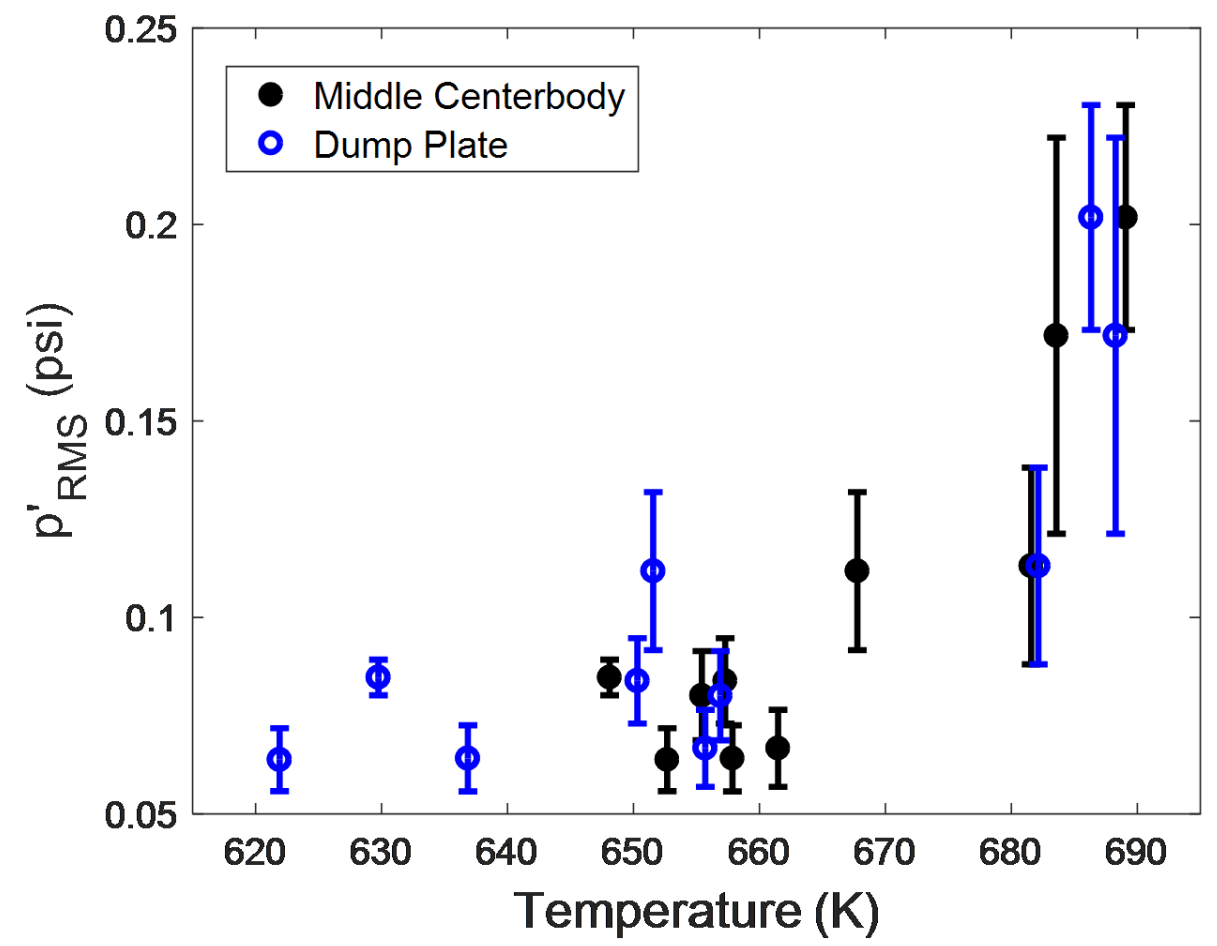

Figure 4: Pressure fluctuation amplitude as a function of centerbody and dump plate temperatures $(1 \mathrm{psi}=6894.76 \mathrm{~Pa})$

\section{Criteria for stable and unstable operation}

The combustor is considered to be unstable when two criteria are satisfied. The first is that the combustor pressure fluctuation amplitude is higher than a threshold value of $0.5 \%$ of the mean combustor pressure. In the current system, where the combustor is run at atmospheric pressure, this value corresponds to around $0.07 \mathrm{psi}(0.48 \mathrm{kPa})$. The second criterion is that a well-defined peak (amplitude-based SNR > 30) exists in the amplitude/power spectrum. Figure 5 
shows amplitude spectra of the combustor pressure fluctuation measured during stable operation and unstable operation plotted on a logarithmic scale. The amplitude of $p_{R M S}^{\prime}$ at the unstable condition is more than an order of magnitude larger than that at the stable condition. A welldefined peak at a frequency of $524 \mathrm{~Hz}$ is also observed in the unstable case.

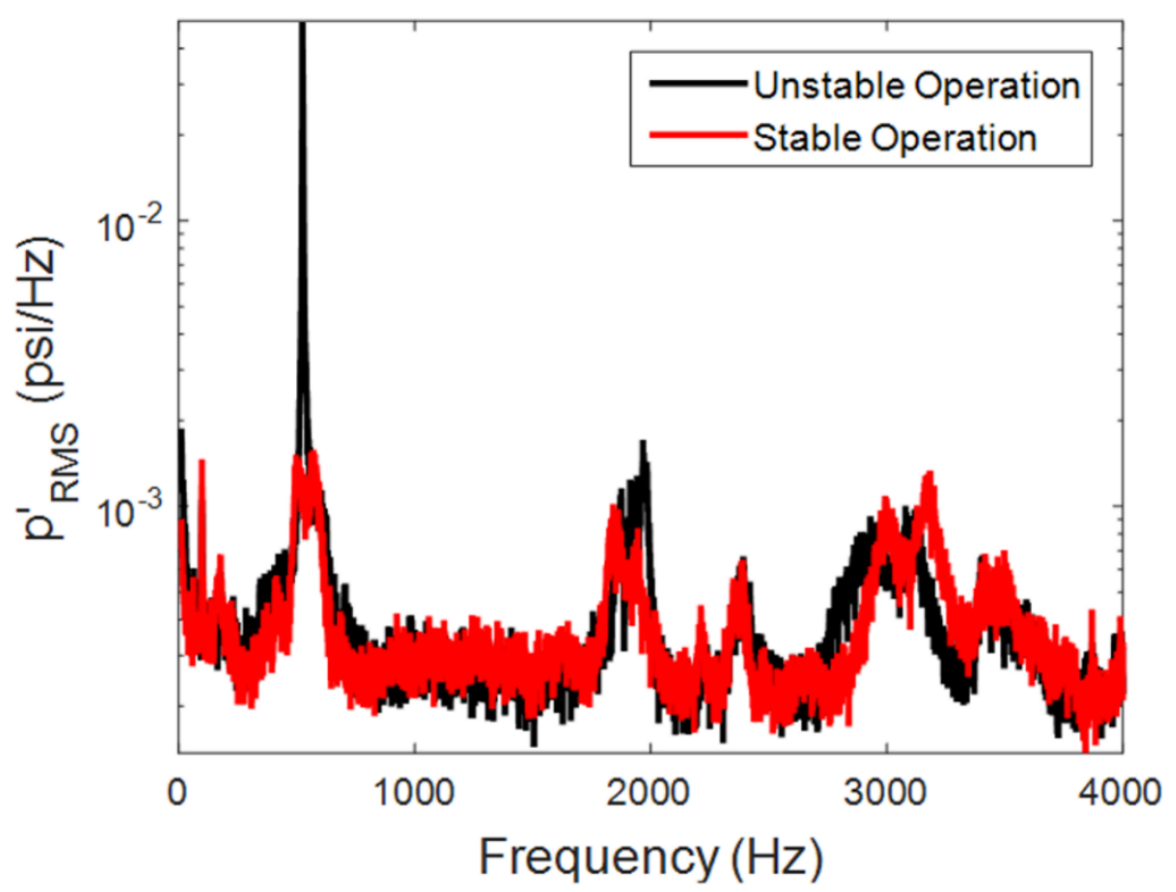

Figure 5: Amplitude spectra of combustor pressure fluctuation (1 psi = 6894.76 Pa)

\section{Operating conditions}

The target operating range for the experiment is as follows: a fixed inlet velocity (U) of $26 \mathrm{~m} / \mathrm{s}$, a fixed inlet temperature $\left(\mathrm{T}_{\text {in }}\right)$ of $473 \mathrm{~K}$, and an equivalence ratio range from 0.65 to 0.73. Transitions between unstable conditions where the flame undergoes a self-excited instability and stable conditions (and vice versa) are achievable in this operating range by changing the equivalence ratio, as evidenced with the trend of the blue data points in the plot of combustor pressure fluctuation $\left(p_{R M S}^{\prime}\right)$ as a function of equivalence ratio in Fig. 6. The error bars denote \pm 1 standard deviation of 16 one second samples. 
When investigating the effect of fuel splits on the transition from unstable to stable operation, test cases were defined in terms of equivalence ratio. At each fuel split test case, the equivalence ratio of the four outer nozzles was kept constant, while the equivalence ratio of the middle nozzle was increased by the injection of additional fuel. A fuel split test case is characterized by the overall equivalence ratio $\left(\varphi_{\text {overall }}\right)$ defined using Eq. (5).

$$
\varphi_{\text {overall }}=\frac{\varphi_{\text {middle }}+4 \cdot \varphi_{\text {outer }}}{5}
$$

where $\varphi_{\text {middle }}$ is the equivalence ratio of the middle nozzle and $\varphi_{\text {outer }}$ is the equivalence ratio of each of the four outer nozzles. The operating points are tabulated in Table 1 . Test case 1 is a baseline case where all five nozzles are fueled equally and the combustor is stable. The combustor experiences a self-excited instability at test case 2, which is suppressed by fuel staging (test cases 3-4 and 5-6). In test cases 3-4, the equivalence ratio of the outer nozzles was kept constant while the middle nozzle equivalence ratio was increased. In test cases 5-6, the overall equivalence ratio was kept constant by decreasing the outer nozzle equivalence ratio while increasing the middle nozzle equivalence ratio.

Table 1: Fuel split test cases

\begin{tabular}{|c|c|c|c|c|c|c|}
\hline Test Case & $\boldsymbol{\varphi}_{\text {outer }}$ & $\boldsymbol{\varphi}_{\text {middle }}$ & $\boldsymbol{\varphi}_{\text {overall }}$ & Percent staging & $\mathbf{p}_{\text {RMS }}(\mathbf{p s i})$ & $\mathbf{p}_{\text {RMS }}(\mathbf{P a})$ \\
\hline $\mathbf{1}$ & 0.65 & 0.65 & 0.65 & 0 & 0.01 & 68.95 \\
\hline $\mathbf{2}$ & 0.70 & 0.70 & 0.70 & 0 & 0.21 & 1447.9 \\
\hline $\mathbf{3}$ & 0.70 & 0.75 & 0.71 & 1.4 & 0.14 & 965.3 \\
\hline $\mathbf{4}$ & 0.70 & 0.86 & 0.73 & 4.4 & 0.02 & 137.9 \\
\hline $\mathbf{5}$ & 0.69 & 0.74 & 0.70 & 1.4 & 0.11 & 758.4 \\
\hline $\mathbf{6}$ & 0.67 & 0.82 & 0.70 & 4.4 & 0.02 & 137.9 \\
\hline
\end{tabular}


The amount of fuel staging can also be characterized by percent staging, which is the percentage of additional fuel flow (relative to the total fuel flow to the combustor) added to the middle nozzle during fuel staging. The red and magenta data points in Fig. 6 show the effect of $1.4 \%$ and $4.4 \%$ staging on combustor pressure fluctuation amplitude. Once again, the error bars denote \pm 1 standard deviation of 16 one second samples.

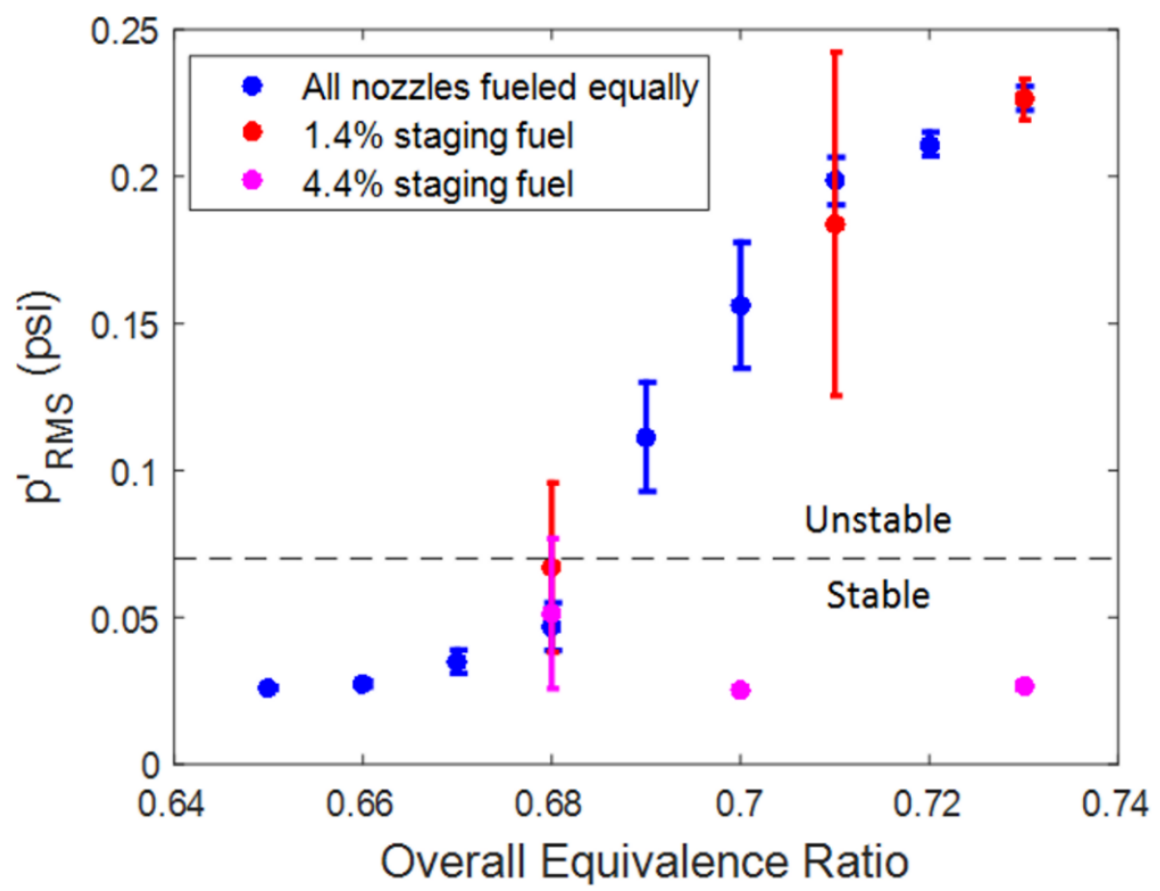

Figure 6: Combustor pressure fluctuation as a function of equivalence ratio at different fuel staging percentages $(1 \mathrm{psi}=6894.76 \mathrm{~Pa})$

Figure 6 shows that introducing a small amount of staging fuel has a minimal effect on the instability amplitude, but can increase its intermittency at some conditions by bringing the combustor closer to the stability boundary. This is evidenced by the large deviation of the pressure fluctuation (larger error bars). Increasing the staging fuel results in the combustor transitioning from unstable to stable operation. Higher levels of staging, $4.4 \%$, are shown to complete suppress the instability in this combustor. 


\section{RESULTS}

\section{The effect of fuel staging on combustor pressure fluctuation}

At the target operating condition, the combustor successfully transitions from unstable (test case 2) to stable operation with increased fuel staging. This is possible for both staging strategies, i.e., increasing overall equivalence ratio (test cases 3-4) and keeping overall equivalence ratio constant (test cases 5-6). Figure 7 shows a plot of $p_{R M S}^{\prime}$ vs. $\varphi_{\text {overall }}$ for the two staging strategies. The test cases with constant overall equivalence ratio are denoted with blue asterisks and the test cases with constant outer nozzle equivalence ratio are denoted with filled black circles.

In both cases, increasing the middle nozzle equivalence ratio suppresses the combustor pressure fluctuation. The equivalence ratio gradient caused by fuel staging could vary the flame's length scale or heat release rate distribution, both which have been shown to affect combustion dynamics in single-nozzle flames $[23,24]$. To better understand the impact of fuel staging on the flame structure of the multi-nozzle flame, line-of-sight chemiluminescence images and flame surface density images obtained from OH-PLIF are analyzed in the next section. 


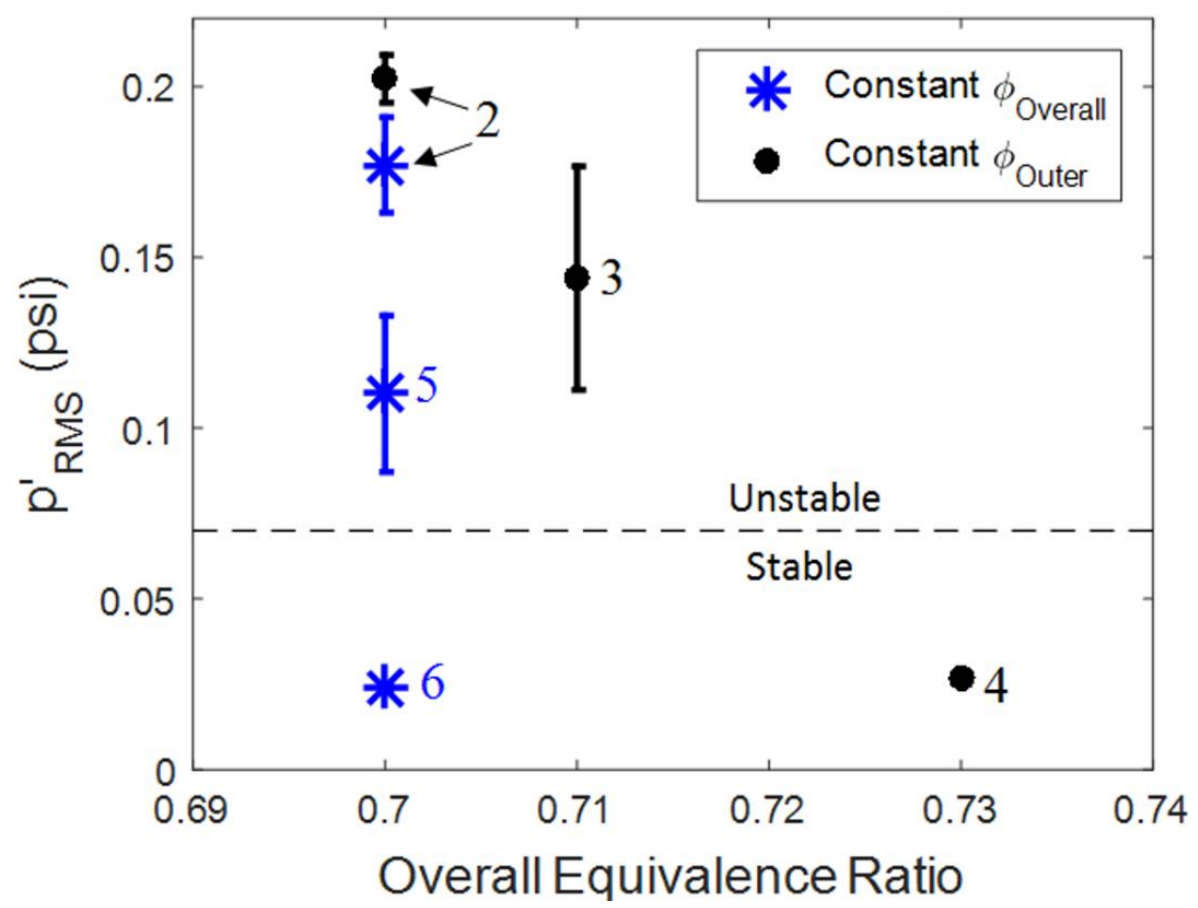

Figure 7: Reduction of Combustor pressure fluctuation amplitude with increased fuel staging $(1 \mathrm{psi}=6894.76 \mathrm{~Pa})$

\section{Analysis of mean flame structure}

Figure 8 shows time-averaged line-of-sight $\mathrm{CH}^{*}$ chemiluminescence images of the multinozzle flame for test cases 1-4. In all test cases, the greatest heat release rate (chemiluminescence) is observed in the region where adjacent flames interact. In this view, the overall flame shape looks very similar in the stable and unstable cases where all five nozzles are fueled equally (Figs. 8a and $8 \mathrm{~b}$ ) with the flame appearing slightly more compact in the unstable case. With the introduction of fuel staging (Figs. 8c-d), the overall flame shape isn't altered significantly in this view, but changes in the chemiluminescence distribution can be observed. Increased fuel staging elevates the chemiluminescence intensity in the interaction region. This is expected since the additional fuel injected to the middle nozzle increases the equivalence ratio of the middle flame and chemiluminescence intensity increases with increased equivalence ratio. 
The outer flame structure is unchanged with fuel staging which suggests that fuel staging suppresses self-excited instabilities through mechanisms that occur in the middle flame and/or the flame-flame interaction region.

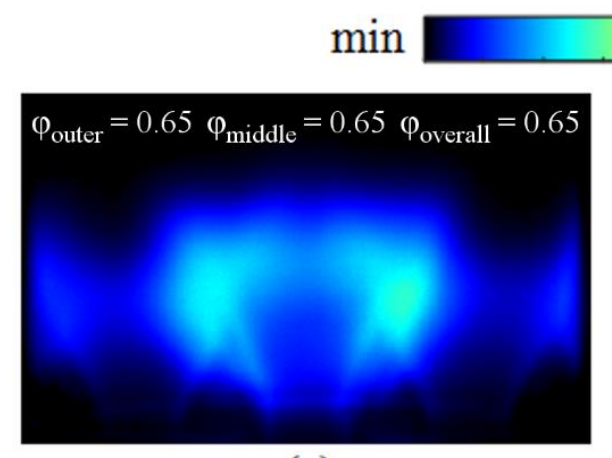

(a)

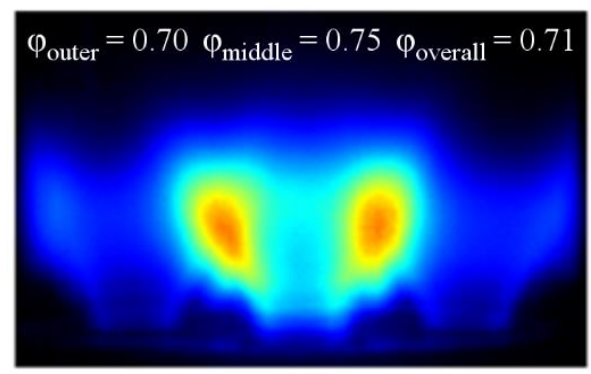

(c)

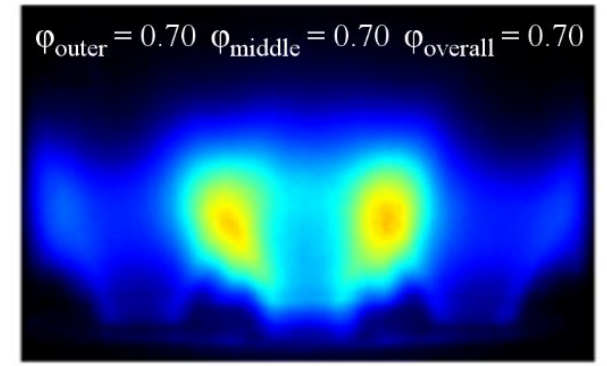

(b)

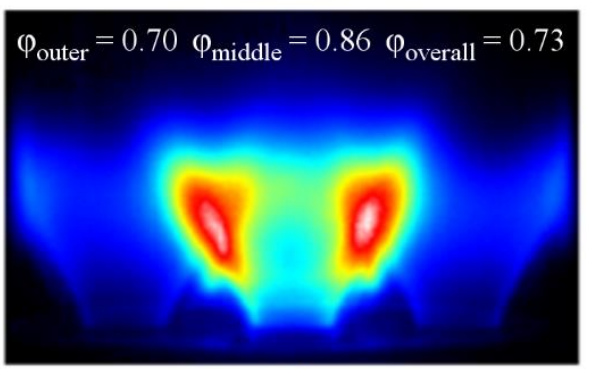

(d)

\section{Figure 8: Time-averaged $\mathrm{CH}^{*}$ chemiluminescence images of the multi-nozzle flame at test}

$$
\text { cases (a) 1, (b) 2, (c) 3, and (d) } 4
$$

Time-averaged FSD images of test cases 1, 2 and 4 are shown in Fig. 9. The solid gray regions in each image show the dump plate location and the hatched gray regions show the centerbody locations. As the flame edges are time-averaged, the flames appear as a "brush", the thickness of which can be used as an indicator of the extent of flame front movement. Figures 9a and $9 \mathrm{~b}$ show data from the test cases when all nozzles of the multi-nozzle flame are fueled equally. In the unstable case (Fig. 9b), secondary flame stabilization in the outer shear layers is observed in the region between the middle and outer flames. In contrast, the flames are stabilized along only the inner shear layer in the stable case (Fig. 9a). This is consistent with previous data 
from single-nozzle flames, which showed a transition from stable to unstable when the flame shape changed from one that was stabilized in the inner shear layer to one that was stabilized in both inner and outer shear layers [25]. Differences in the outer branch of the outer flame are negligible between the stable and the unstable cases, which indicate that the transition to unstable behavior may be, in part, caused by changes in flame structure in the flame-flame interaction. Another observation is that the flame brushes are thicker in the unstable case, which is indicative of increased motion of the flame fronts. In the case when the flame is stable due to fuel staging (Fig. 9c), the flame is still stabilized on both the inner and outer shear layers with the only difference being that the flame brushes are thinner than the unstable case (Fig. 9b), which is indicative of suppressed flame front movement. The fact that the two stable cases (Figs. 9a and 9c) exhibit differences in flame structure suggests that they are stable through different mechanisms. To better understand those mechanisms, time-resolved line-of-sight chemiluminescence images are analyzed in the next section.

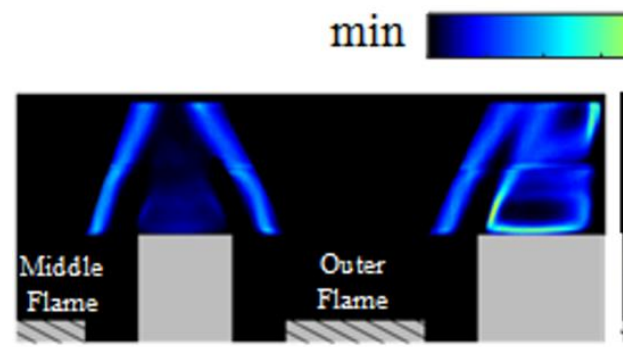

(a)

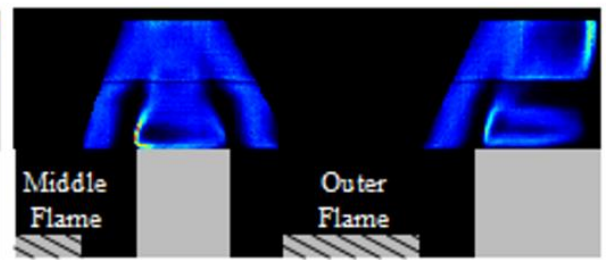

(b)

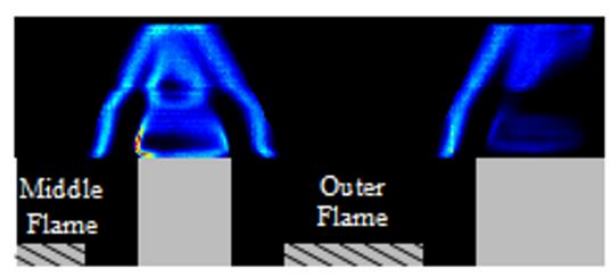

(c)

Figure 9: Time-averaged flame surface density images of the multi-nozzle flame at test cases (a) 1, (b) 2, and (c) 4 


\section{Analysis of fluctuating flame structure}

RMS and phase images obtained from the line-of-sight chemiluminescence image (introduced in the data processing section) and the Rayleigh integral distribution within the flame are analyzed in this section in order to understand the unsteady flame structure during stable and unstable operating conditions.

Figure 10 shows normalized RMS images of test cases $1-4$. The maximum pixel value of each image has been adjusted to enhance features the flame brush and is labeled in the top left corner of each image. In the test case when all five nozzles are fueled equally and the combustor is unstable (Fig. 10b), large fluctuations in heat release rate are observed at the downstream portion of the flame. These heat release rate fluctuations are most likely due to flame tip movement caused by either vortices shed along the shear layers interacting with the flame or bulk movement of the flame due to acoustic perturbations. This cannot be definitively determined from line-of-sight images alone, and further analysis with more advanced diagnostics is required to further investigate these phenomena.

When a small amount of staging fuel is introduced, these fluctuations persist (Fig. 10c), but they become suppressed as fuel staging is increased further (Fig. 10d). In the highest staging case, the fluctuations are not completely suppressed and some very low amplitude fluctuations are still observed. In particular, it is instructive to compare Figs. 10a and 10d, where the pressure fluctuation amplitudes are both far below the instability limit. The flame structure and patterns of heat release rate oscillations are still evident in test case 4, whereas no such structure is seen in test case 1 , despite both being "stable" cases. 


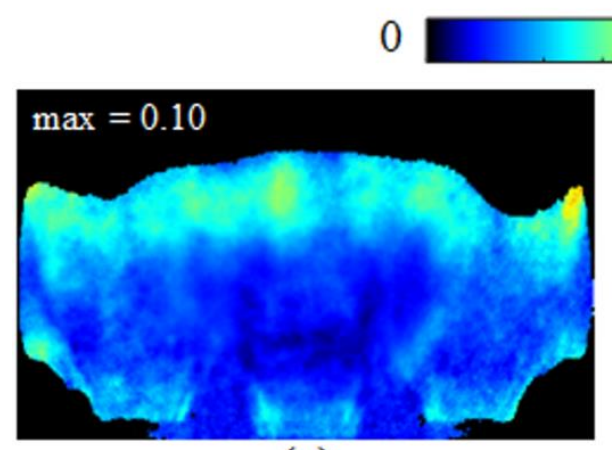

(a)

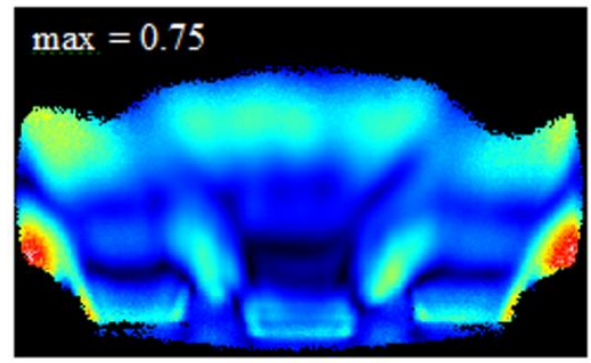

(c)

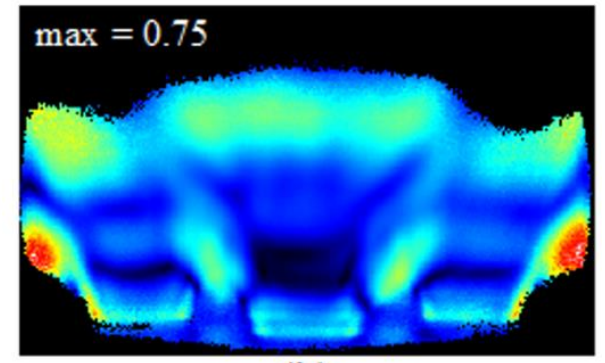

(b)

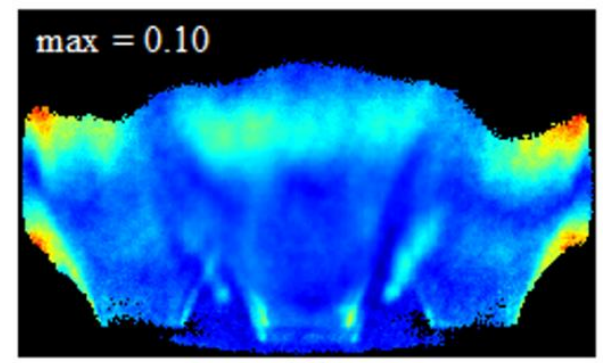

(d)

Figure 10: Normalized RMS images of the multi-nozzle flame at test cases (a) 1, (b) 2, (c) 3, and (d) 4

Figure 11 shows phase images at test cases 1-4. During the baseline test case where the combustor is stable (Fig. 11a), the phase distribution is incoherent throughout the flame. In contrast, when the flame is subject to a self-excited instability (Fig. 11b), the phase distribution becomes more coherent. As the downstream distance from the flame anchoring location increases (bottom to top of images), the change in phase is continuous, which suggests that the flame response mechanisms are convective. Additionally, the phase is relatively constant across the radial direction at a given downstream location, indicating that the disturbance is affecting all five flames in-phase. 


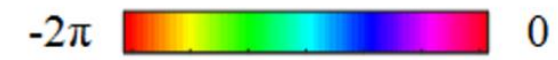

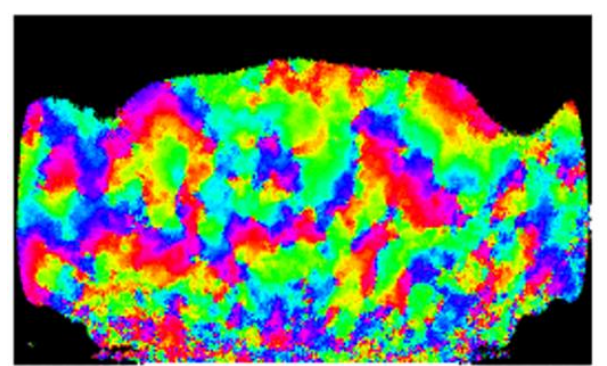

(a)

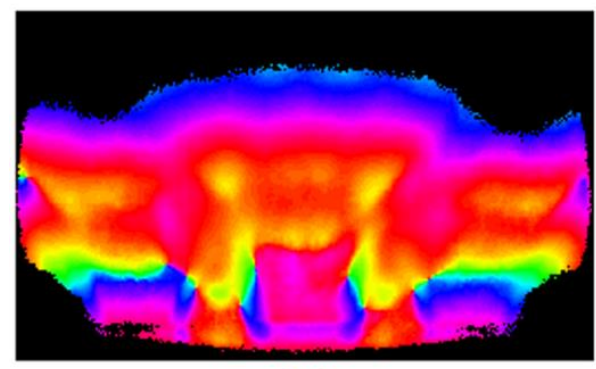

(c)

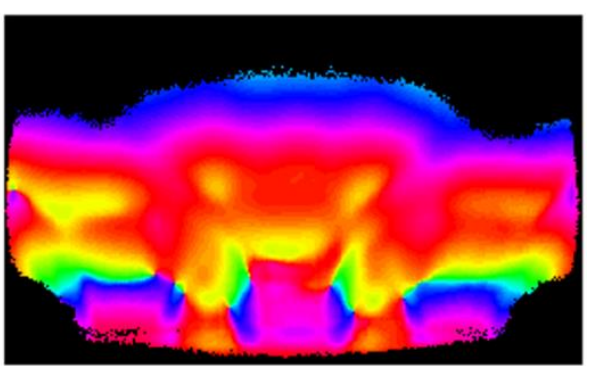

(b)

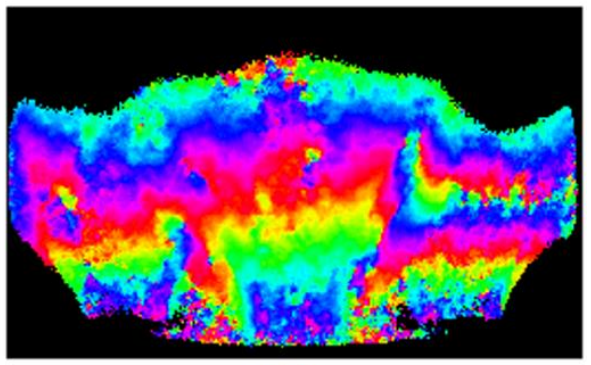

(d)

Figure 11: Phase images of the multi-nozzle flame at test cases (a) 1, (b) 2, (c) 3, and (d) 4

As fuel staging is increased, the nature of this phase relationship is altered, especially at the interaction regions. At the highest staging condition (Fig. 11d), convective mechanisms still exist but are far less coherent than in the unstable case. Additionally, jumps in the phase are present between adjacent flames, suggesting that there may be a phase offset that is the cause of the instability suppression. This is particularly meaningful when considering the presence of coherent regions of high amplitude in the RMS image of test case 4 (Fig. 10d), and the lack of coherent regions in the RMS image of test case 1 (Fig. 10a).

Putting together the RMS and phase information suggests that test case 1 and test case 4 are thermoacoustically stable for different reasons. In test case 1, there is no coherent motion in the flow field, as suggested by the phase, and the RMS is low; the Rayleigh criterion has not been met globally. However, in test case 4, there are still coherent oscillations in the flow field, 
but the phase information suggests that there is a phase cancellation phenomenon between different regions of the flame that causes the overall system to be thermoacoustically stable.

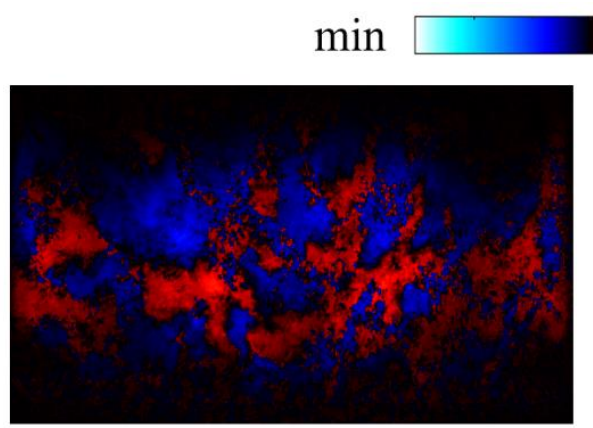

(a) $\max$

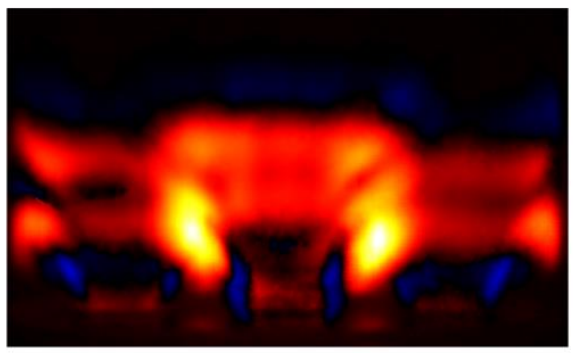

(b)

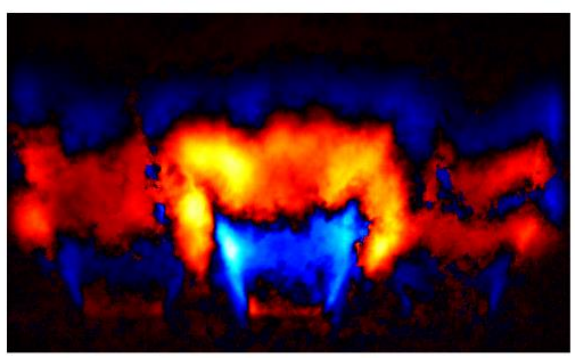

(c)

Figure 12: Rayleigh integral distribution images of the multi-nozzle flame at test cases (a)

$$
\text { 1, (b) } 2 \text {, and (c) } 4
$$

Figure 12 shows Rayleigh integrals for test cases 1, 2, and 4. The hot-cold colormap used denotes areas where the heat release rate fluctuations are out-of-phase with pressure fluctuations (negative Rayleigh integral) as shades of blue and areas where heat release rate fluctuations are in-phase with pressure fluctuations (positive Rayleigh integral) as shades of red. The maximum pixel value of each image has been adjusted to highlight features within the flame brush. Figure 12a shows the Rayleigh integral distribution for the baseline test case where the flame is stable and all five nozzles are fueled equally. The regions of positive and negative Rayleigh integral appear to be almost equally distributed with no coherent pattern observed. This is expected as the fluctuations in this case are mostly due to turbulence in the combustor flow field. When all five 
nozzles are fueled equally and the combustor is unstable (test case 2), large regions where the Rayleigh integral is positive are observed especially in the flame-flame interaction regions (Fig. 12b). In comparing this to the RMS image in Fig. 10b, it is apparent that these regions correspond to the regions of highest fluctuation amplitude. This indicates that the regions of the flame with the greatest heat release rate fluctuation positively couple with the combustor pressure fluctuations.

As staging fuel is introduced, the regions of high heat release fluctuation, especially the flame-flame interaction region now oscillate out-of-phase with the pressure fluctuation as evidenced by those regions appearing blue in Fig. 12c. This combined with the fact that coherent bands of positive and negative Rayleigh integral still exist within the flame brush further suggest that the mechanism by which fuel staging suppresses instabilities is by altering the phase of convective disturbances that travel along the flame front.

\section{SUMMARY AND CONCLUSIONS}

An experimental study of the effects of fuel staging on the flame stabilization and heat release rate distribution (referred to as flame structure), and self-excited instability characteristics was conducted in a natural gas-fueled multi-nozzle can combustor where fuel staging was achieved by injecting additional fuel at the middle nozzle. At the operating conditions considered, self-excited instabilities were suppressed with fuel staging: in both cases where overall equivalence ratio is increased by staging (by only increasing the fuel flow rate to the middle nozzle) as well as cases where overall equivalence ratio is kept constant while staging (by simultaneously decreasing the fuel flow rate of the outer nozzles while increasing the fuel flow rate to the middle nozzle). 
From the analysis of time-averaged line-of-sight $\mathrm{CH}^{*}$ chemiluminescence images, it is evident that fuel staging changes the heat release rate distribution in the region where adjacent flames interact. Furthermore, time-averaged flame surface density images obtained using $\mathrm{OH}-$ PLIF showed that when all five nozzles were fueled equally, the flame stabilized in both the inner and outer shear layers between the middle and outer flames when the flame was unstable. In contrast, flame stabilization only occurred in the inner shear layer of this region when the flame was stable. With increased fuel staging, these "secondary" flame structures in the outer shear layer remained, suggesting that a different mechanism acts to stabilize the flame when staging fuel is introduced.

RMS images obtained from line-of-sight $\mathrm{CH}^{*}$ chemiluminescence images show large fluctuations in heat release rate in the downstream portion of the flame, which are most likely due to flame tip movement. With increased fuel staging, these fluctuations are not completely suppressed in that the patterns of heat release rate oscillations still exist but at much lower amplitudes. The phase distribution of the line-of-sight chemiluminescence images shows that when the flame is unstable, the phase varies monotonically with downstream distance from the flame anchoring location suggesting that the flame response mechanisms are convective. Additionally, the radial variation of phase at a given downstream location is minimal, indicating that the disturbance is affecting all five flames in-phase. As fuel staging is increased, the nature of this phase relationship is altered, especially at the interaction regions. Convective mechanisms still exist but are far less coherent than in the unstable case.

Rayleigh integral distribution images show that as fuel staging is increased, the altered phase of the convective disturbances causes regions of the flame that previously were in-phase with the pressure fluctuation to oscillate out-of-phase, thus suppressing the instability. Since the 
added staging fuel is technically premixed, this phase difference could be due to a combination of velocity fluctuations or equivalence ratio fluctuations. Further study is needed to determine the relative contribution of each mechanism.

\section{ACKNOWLEDGEMENTS}

The authors would like to acknowledge Stephen Peluso from Penn State's Center for Combustion, Power and Propulsion, and Keith McManus, Tony Dean, and Fei Han from GE Global Research for their suggestions and insight on this work. The authors also want to thank Mr. Larry Horner for fabricating many of the components of the multi-nozzle combustor. This work was funded by the U.S. Department of Energy under award number DEFE0025495 and contract monitor Mark Freeman. The high-speed LIF system used in this study was provided through the College of Engineering Instrumentation Grant Program and the Department of Mechanical and Nuclear Engineering at Penn State.

\section{REFERENCES}

[1] Rayleigh, J. W. S., 1878, "The Explanation of Certain Acoustical Phenomena," Nature, 18(455), pp. 319-321.

[2] Huang, Y., and Yang, V., 2009, "Dynamics and Stability of Lean-Premixed Swirl-Stabilized Combustion," Prog. Energy Combust. Sci., 35(4), pp. 293-364.

[3] Ducruix, S., Schuller, T., Durox, D., and Candel, S., 2003, "Combustion Dynamics and Instabilities: Elementary Coupling and Driving Mechanisms," J. Propul. Power, 19(5), pp. 722-734.

[4] Candel, S., Durox, D., Schuller, T., Bourgouin, J.-F., and Moeck, J. P., 2014, "Dynamics of Swirling Flames," Annual Review of Fluid Mechanics, 46(1), pp. 147-173. 
[5] Külsheimer, C., and Büchner, H., 2002, "Combustion Dynamics of Turbulent Swirling Flames," Combust. Flame, 131(1), pp. 70-84.

[6] Balachandran, R., Ayoola, B., Kaminski, C., Dowling, A., and Mastorakos, E., 2005, "Experimental Investigation of the Nonlinear Response of Turbulent Premixed Flames to Imposed Inlet Velocity Oscillations," Combust. Flame, 143(1), pp. 37-55.

[7] Palies, P., Durox, D., Schuller, T., and Candel, S., 2010, "The Combined Dynamics of Swirler and Turbulent Premixed Swirling Flames," Combust. Flame, 157(9), pp. 16981717.

[8] Bunce, N. A., Quay, B. D., and Santavicca, D. A., 2014, "Interaction between Swirl Number Fluctuations and Vortex hedding in a Single-Nozzle Turbulent Swirling Fully-Premixed Combustor," ASME J. Eng. Gas Turbines Power, 136(2), p. 021503.

[9] Steinberg, A. M., Arndt, C. M., and Meier, W., 2013, "Parametric Study of Vortex Structures and Their Dynamics in Swirl-Stabilized Combustion," Proc. Combust. Inst., 34(2), pp. $3117-3125$.

[10] Acharya, V. S., Shin, D.-H., and Lieuwen, T., 2013, "Premixed Flames Excited by Helical Disturbances: Flame Wrinkling and Heat Release Oscillations," J. Propul. Power, 29(6), pp. $1282-1291$.

[11] Szedlmayer, M. T., 2013, "An Experimental Study of the Velocity-Forced Flame Response of a Lean-Premixed Multi-Nozzle Can Combustor for Gas Turbines," Ph.D., The Pennsylvania State University, University Park, PA.

[12] Fanaca, D., Alemela, P., Hirsch, C., and Sattelmayer, T., 2010, "Comparison of the Flow Field of a Swirl Stabilized Premixed Burner in an Annular and a Single Burner Combustion Chamber," ASME J. Eng. Gas Turbines Power, 132(7), p. 071502. 
[13] Samarasinghe, J., Peluso, S. J., Quay, B. D., and Santavicca, D. A., 2016, "The ThreeDimensional Structure of Swirl-Stabilized Flames in a Lean Premixed Multinozzle Can Combustor," ASME J. Eng. Gas Turbines Power, 138(3), p. 031502.

[14] McManus, K., Poinsot, T., and Candel, S., 1993, "A Review of Active Control of Combustion Instabilities," Prog. Energy Combust. Sci., 19(1), pp. 1-29.

[15] Bulat, G., Skipper, D., McMillan, R., and Syed, K., 2007, "Active Control of Fuel Splits in Gas Turbine Dle Combustion Systems,"ASME Paper No. GT2007-27266.

[16] Lefebvre, A. H., and Ballal, D. R., 2010, Gas Turbine Combustion, CRC Press.

[17] Davis, L. B., and Black, S. H., 1995, "Dry Low Nox Combustion Systems for Ge HeavyDuty Gas Turbines," GE Power Systems, Schenectady, NY.

[18] Szedlmayer, M. T., Quay, B. D., Samarasinghe, J., De Rosa, A., Lee, J. G., and Santavicca, D. A., 2011, "Forced Flame Response of a Lean Premixed Multi-Nozzle Can Combustor,"ASME Paper No. GT2011-46080.

[19] Lee, J. G., and Santavicca, D. A., 2003, "Experimental Diagnostics for the Study of Combustion Instabilities in Lean Premixed Combustors," J. Propul. Power, 19(5), pp. 735750.

[20] Nori, V., and Seitzman, J., 2008, "Evaluation of Chemiluminescence as a Combustion Diagnostic under Varying Operating Conditions,"AIAA Paper No. 2008-953.

[21] Oppenheim, A. V., and Schafer, R. W., 2010, Discrete-Time Signal Processing, Pearson Higher Education.

[22] Foley, C., 2015, "Attachment Point Characteristics and Modeling of Shear Layer Stabilized Flames in an Annular, Swirling Flow Field," Ph.D., Georgia Institute of Technology, Atlanta, GA. 
[23] Kim, K. T., Lee, J. G., Lee, H. J., Quay, B. D., and Santavicca, D. A., 2010, "Characterization of Forced Flame Response of Swirl-Stabilized Turbulent Lean-Premixed Flames in a Gas Turbine Combustor," ASME J. Eng. Gas Turbines Power, 132(4), p. 041502.

[24] Kim, D., Lee, J. G., Quay, B. D., Santavicca, D. A., Kim, K., and Srinivasan, S., 2010, "Effect of Flame Structure on the Flame Transfer Function in a Premixed Gas Turbine Combustor," ASME J. Eng. Gas Turbines Power, 132(2), p. 021502.

[25] Taamallah, S., LaBry, Z. A., Shanbhogue, S. J., and Ghoniem, A. F., 2015, "ThermoAcoustic Instabilities in Lean Premixed Swirl-Stabilized Combustion and Their Link to Acoustically Coupled and Decoupled Flame Macrostructures," Proc. Combust. Inst., 35(3), pp. 3273-3282. 